\title{
Relating basic properties of bright early-type dwarf galaxies to their location in Abell 901/902
}

F. D. Barazza ${ }^{1}$, C. Wolf ${ }^{2}$, M. E. Gray ${ }^{3}$, S. Jogee ${ }^{4}$, M. Balogh ${ }^{5}$, D. H. McIntosh ${ }^{6}$, D. Bacon ${ }^{7}$, M. Barden ${ }^{8}$, E. F. Bell ${ }^{9}$, A. Böhm ${ }^{8,15}$, J. A. R. Caldwell ${ }^{10}$, B. Häussler ${ }^{3}$, A. Heiderman ${ }^{4}$, C. Heymans ${ }^{11}$, K. Jahnke ${ }^{9}$, E. van Kampen ${ }^{12}$, K. Lane ${ }^{3}$, I. Marinova ${ }^{4}$, K. Meisenheimer ${ }^{8}$, C. Y. Peng ${ }^{13}$, S. F. Sanchez ${ }^{14}$, A. Taylor ${ }^{10}$, L. Wisotzki ${ }^{15}$, and X. Zheng ${ }^{16}$

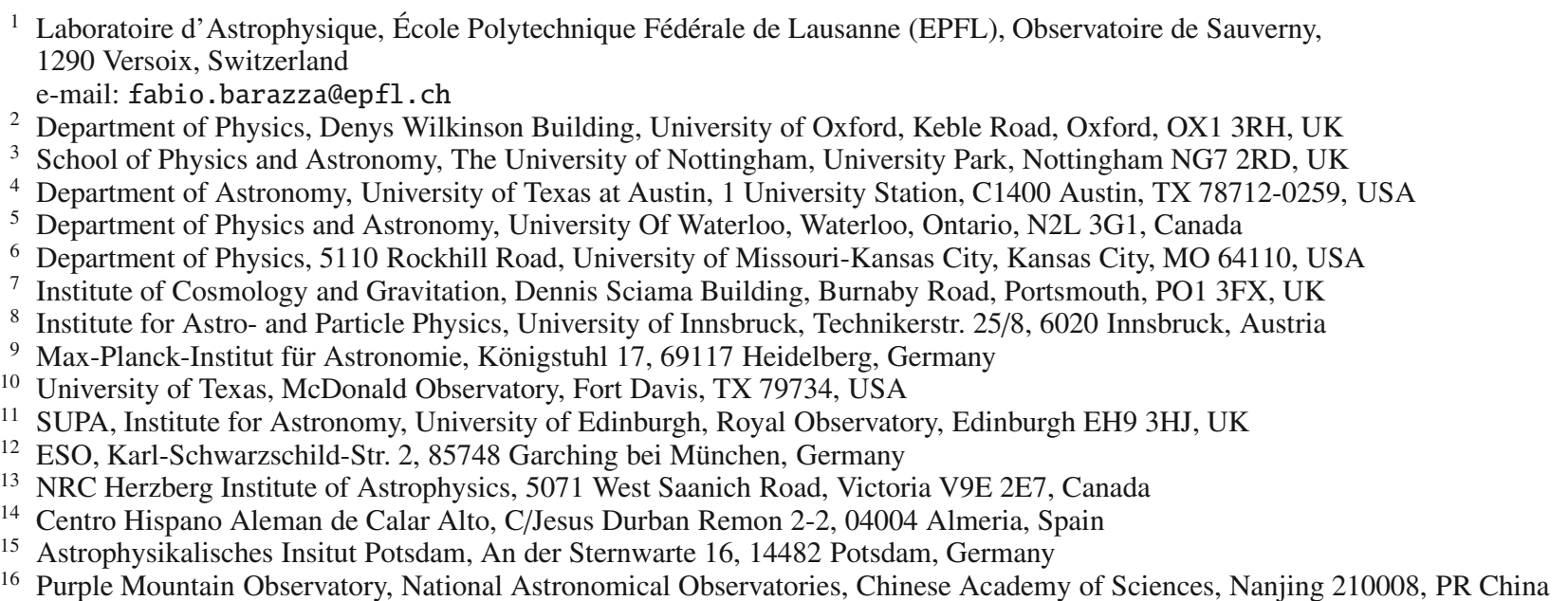

Received 18 June 2009 / Accepted 16 October 2009

ABSTRACT

\begin{abstract}
We present a study of the population of bright early-type dwarf galaxies in the multiple-cluster system Abell 901/902. We use data from the STAGES survey and COMBO-17 to investigate the relation between the color and structural properties of the dwarfs and their location in the cluster. The definition of the dwarf sample is based on the central surface brightness and includes galaxies in the luminosity range $-16 \geq M_{B} \gtrsim-19$ mag. Using a fit to the color magnitude relation of the dwarfs, our sample is divided into a red and blue subsample. We find a color-density relation in the projected radial distribution of the dwarf sample: at the same luminosity dwarfs with redder colors are located closer to the cluster centers than their bluer counterparts. Furthermore, the redder dwarfs are on average more compact and rounder than the bluer dwarfs. These findings are consistent with theoretical expectations assuming that bright early-type dwarfs are the remnants of transformed late-type disk galaxies involving processes such as ram pressure stripping and galaxy harassment. This indicates that a considerable fraction of dwarf elliptical galaxies in clusters are the results of transformation processes related to interactions with their host cluster.
\end{abstract}

Key words. galaxies: dwarf - galaxies: formation - galaxies: evolution - galaxies: clusters: individual: Abell 901/902 galaxies: structure

\section{Introduction}

Early-type dwarf galaxies appear to be spheroidal stellar systems with no signs of ongoing star formation, dust content, or substructures, except a compact high surface brightness nucleus in the center, which is found in a large fraction of early-type dwarfs (Caldwell 1983; Binggeli \& Cameron 1991; Ryden et al. 1999; Barazza et al. 2003; Lisker et al. 2006). Whether earlytype dwarfs constitute a genuine class of galaxies or are merely the counterparts of giant elliptical galaxies at lower luminosities is a matter of ongoing debate. The interpretation of earlytype dwarfs as smaller and fainter versions of giant ellipticals is based on the continuity of some structural parameters (Jerjen \& Binggeli 1997; Graham \& Guzmán 2003; Trujillo et al. 2004; Gavazzi et al. 2005; Ferrarese et al. 2006), while the dichotomy picture rests upon the assumption that early-type giants and dwarfs have different formation origins (merger vs. transformation; Wirth \& Gallagher 1984; Kormendy 1985; Kormendy et al. 2009). In the latter case early-type dwarfs are often referred to as spheroidals, in order to distinguish them from ellipticals. We refer to our sample of early-type dwarfs as dwarf ellipticals (dEs), without giving preference to either of the two interpretations.

One of the most important aspects of dEs is that they are almost exclusively located in regions of high galaxy density. In fact, they can be regarded as the typical galaxy type in galaxy clusters, where they numerically dominate the total galaxy population (Binggeli et al. 1985; Ferguson \& Sandage 1989; Ferguson \& Binggeli 1994). On the other hand they seem to be almost absent in low density regions outside of clusters and galaxy groups (Binggeli et al. 1990; Mateo 1998; Haines et al. 2007). Therefore, the formation and evolution of dEs seems to be intimately connected to their environment. The most likely 
processes believed to be dominant in high density environments are ram pressure stripping (Gunn \& Gott 1972; Lin \& Faber 1983; Abadi et al. 1999; van Zee et al. 2004), tidal shaking or stirring (Mayer et al. 2001b), and galaxy harassment (Moore et al. 1998; Mastropietro et al. 2005). These processes can strongly affect infalling galaxies causing substantial mass loss, the termination of star formation, probably preceded by a final starburst, and transform the galaxies morphologically (e.g., Boselli \& Gavazzi 2006). If the structure and photometric properties of dEs are due to such transformation processes, the progenitors might be significantly different. On the other hand, there also remains the possibility that a certain fraction of dEs belong to the primordial cluster galaxy population and never suffered from transformations.

In order to connect the formation of dEs to their environment, observational indicators have to be found, which are very likely to be caused by interactions with the environment. One promising route is to identify properties which the dEs may have inherited from their progenitors, and which have not been erased completely during the transformation. The hidden disk, spiral, and bar features discovered in a number of dEs in different clusters are believed to be such remnant structures (Jerjen et al. 2000; Barazza et al. 2002; Graham et al. 2003; De Rijcke et al. 2003; Lisker et al. 2006). These structures are only visible, when image processing methods such as unsharp masking or model subtractions are applied, and indicate that the dEs are the products of transformations caused by the interactions of disk galaxies with the cluster and its members. Supporting this picture is the observation that some dEs themselves (De Rijcke et al. 2001; Pedraz et al. 2002; Geha et al. 2003; van Zee et al. 2004) or their globular cluster systems are rotating (Beasley et al. 2006, 2009), which indicates their origin as disky systems.

Another indication connecting the origin of dEs to their environment are relations between properties of the $\mathrm{dE}$ population and measures of the local galaxy density. Such relations are expected according to simulations of transformation processes in a cluster environment (Moore et al. 1998; Mastropietro et al. 2005; Tonnesen et al. 2007). Observational indications for corresponding properties of $\mathrm{dE}$ populations in specific galaxy clusters have been found in a number of studies, using different approaches. Secker (1996) found a significant color gradient in the projected radial distribution of a sample of $\sim 250 \mathrm{dEs}$ in the Coma cluster. This color gradient was interpreted as a metallicity gradient caused by the confinement pressure of the intracluster gas (Babul \& Rees 1992). A similar result was reported by Rakos \& Schombert (2004), who found nucleated dEs (dENs) to be redder than non-nucleated dEs. The former are known to exhibit a stronger clustercentric concentration than the latter (van den Bergh 1986; Binggeli et al. 1987) resulting again in a color gradient. Based on the different color properties of dENs they were also found to be on average 5 Gyr older than dEs (Rakos \& Schombert 2004). A different approach was chosen by Michielsen et al. (2008) and Smith et al. (2009), who used spectroscopic observations of dEs in different clusters to find an age gradient in the sense that older dEs are located closer to the cluster centers than their younger counterparts. Such gradients alone do not prove an environmental transformation, but are predicted by models of galaxy interactions in clusters and can therefore be compared quantitatively with these predictions.

In general, the color or age gradients found are rather shallow and the scatters of the individual points are large. We try to improve on this situation with the present study. We use HST/ACS images from the Space Telescope A901/902 Galaxy Evolution Survey (STAGES, Gray et al. 2009) and spectral energy distribution (SED) fits from COMBO-17 (Wolf et al. 2003) to investigate the color and structural properties of the $\mathrm{dE}$ population and their relation to the cluster environment. Our sample of 470 early-type cluster galaxies also includes giant ellipticals, which we use as a comparison sample. The analysis is based on color, central surface brightness, Sérsic index, and concentration index. In Sect. 2 we describe the data used and the definition of the sample. In Sects. 3-5 we discuss the color-magnitude relation of the sample, the distribution of the galaxies in the cluster region, and the compactness of the objects, respectively. A discussion follows in Sect. 6 and a summary in Sect. 7. Throughout the paper, we assume a flat cosmology with $\Omega_{M}=1-\Omega_{\Lambda}=0.3$ and $H_{0}=70 \mathrm{~km} \mathrm{~s}^{-1} \mathrm{Mpc}^{-1}$.

\section{Sample selection from STAGES}

\subsection{The data from the STAGES survey}

STAGES is a multiwavelength survey aimed at the study of galaxy formation and evolution in different environments. It covers an area of $0.5^{\circ} \times 0.5^{\circ}$ centered on the multiple-cluster system Abell 901(a,b)/902. The multiple-cluster system A901/902 at $z=0.165$ consists of four galaxy accumulations of different sizes: three clusters and one group (Gray et al. 2002). The principal dataset used in our study is the 80-orbit HST ACS F606W mosaic, which provides images with a scale of 0. .03/pixel and a point spread function (PSF) of 0. ' 1 , corresponding to $\sim 282 \mathrm{pc}$ at $z=0.165$. For all galaxies photometric redshifts are available from COMBO-17 (Wolf et al. 2003, 2005) with $\delta z /(1+z) \sim 0.007-0.022$ for our sample (Gray et al. 2009). Supplementary to the optical data dark matter maps from weak lensing (Heymans et al. 2008), total star formation rates based on UV and Spitzer observations (Bell et al. 2005), and stellar masses (Borch et al. 2006) are available for the cluster field.

In addition to the multiwavelength dataset described above, the STAGES imaging data were processed with the data pipeline GALAPAGOS (Galaxy Analysis over Large Areas: Parameter Assessment by GALFITting Objects from SExtractor; Barden 2009 , in prep.). Part of this pipeline is an analysis of the surface brightness profiles of the galaxies based on GALFIT (Peng et al. 2002), which provides structural parameters such as effective radii $\left(r_{\mathrm{e}}\right)$ and Sérsic indices $(n)$ for all galaxies. The latter is used in our study as a measure of the compactness of our galaxies. In addition, we also use the CAS concentration index $C$ (Bershady et al. 2000) derived using the CAS code (Conselice et al. 2000, 2003) by Heiderman et al. (2009). See Gray et al. (2009) for a detailed description of the entire data reduction and processing work.

We are only interested in cluster galaxies and our initial sample is based on the general definition of the cluster sample applied to the STAGES catalog (Gray et al. 2009). The membership criterion is based on the photometric redshifts. Galaxies with $z_{\text {cluster }}-\Delta z<z_{\text {galaxy }}<z_{\text {cluster }}+\Delta z$ are considered to be cluster members, where $\Delta z$ depends on the total apparent $R$ magnitude and is defined as

$$
\Delta z(R)=\sqrt{0.015^{2}+0.0096525^{2}\left(1+10^{0.6\left(R_{\mathrm{TOT}}-20.5\right)}\right)}
$$

This photo- $z$ width assures a completeness of $>90 \%$ down to $R_{\text {Vega }}=24$ mag (for details see Gray et al. 2009). This definition results in a cluster sample of 1990 galaxies. Our final definition of the early-type cluster members and the subsample of dwarf galaxies is described below. 

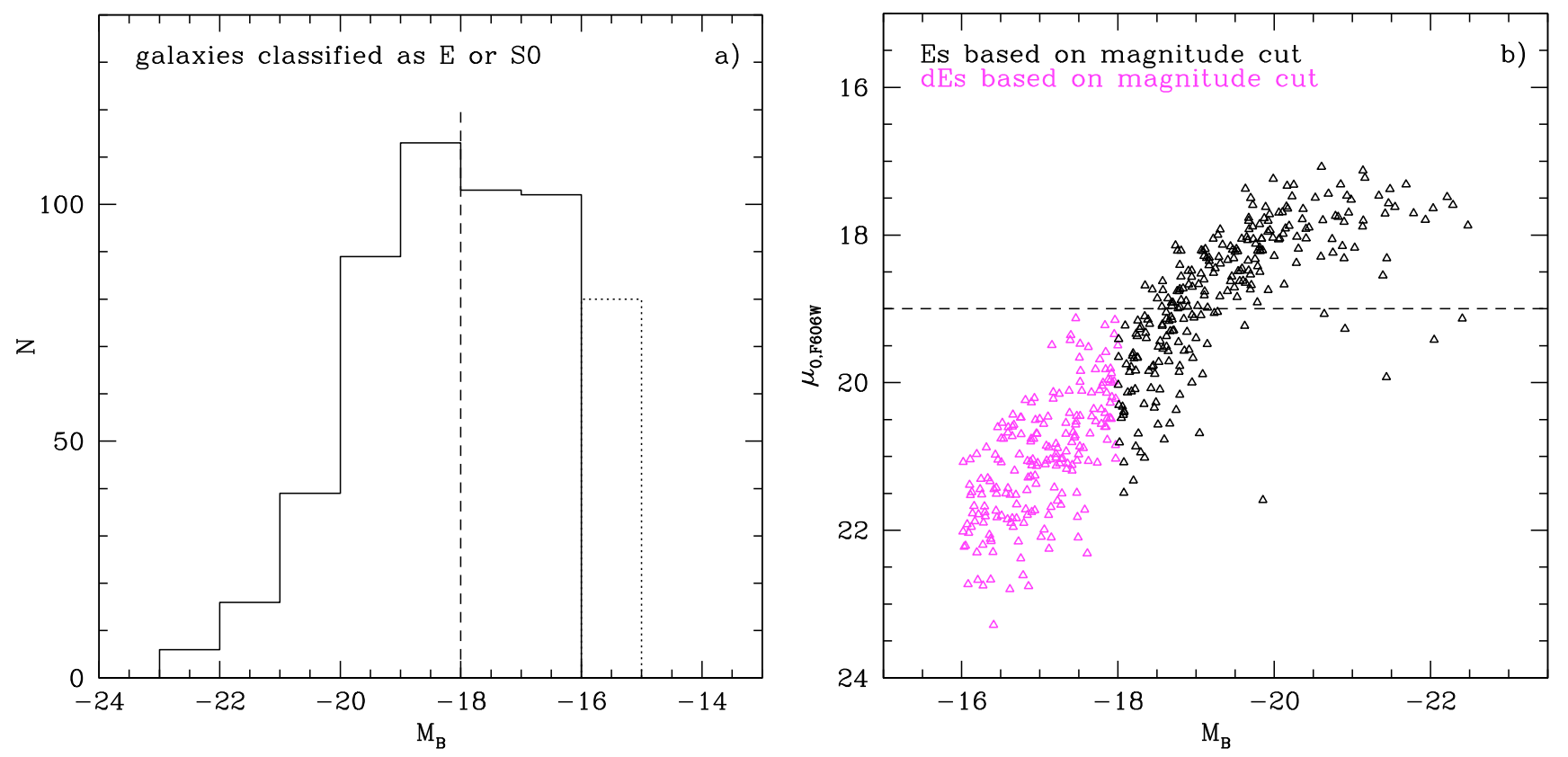

Fig. 1. a) The $M_{B}$ distribution of all early-type galaxies (solid+dotted histogram). The solid histogram shows the sample considered in our study. The dashed line indicates a possible separation between giants and dwarfs. b) Absolute $B$-band magnitude versus central surface brightness measured on the $F 606 \mathrm{~W}$-band images within the central $\sim 420 \mathrm{pc}$ of the galaxies. The dashed line indicates a possible separation between giants and dwarfs.

\subsection{Visual classifications}

All galaxies in the final STAGES catalog with $z<0.4$ and $R<$ $23.5 \mathrm{mag}$ have been visually classified (5090 objects in total). The classification was performed by seven co-authors (AA-S, FDB, MEG, KJ, KL, DHM, CW) making sure that each galaxy was inspected by at least three classifiers. Thus, not all galaxies have been classified by all classifiers, but for each galaxy at least three independent classifications are available. Each galaxy was assigned a Hubble type, including the barred subclasses, representing an average of all available classifications. The agreement among the classifiers was in general very good and we base our selection of the parent sample of early-type galaxies on this classification.

\subsection{The definition of the $d E$ sample}

Based on the cluster sample of 1990 galaxies and their visual classification we define our sample of dEs. First, we select all galaxies classified as E or S0. Since the distinction between Es and S0s is rather difficult, particularly for the fainter dwarf early-type galaxies, we match the E and S0 classes and obtain a sample of 550 early-type galaxies. We note that visual types are only available for 1713 galaxies, since only objects with $R<23.5 \mathrm{mag}$ have been visually classified. This concerns galaxies with $M_{B} \gtrsim-15.5 \mathrm{mag}$, which would be excluded anyway (see below). As shown in Gray et al. (2009, Fig. 14) the contamination by field galaxies increases sharply for objects with $R>22$ mag. We therefore apply a lower magnitude cut at $M_{B} \leq-16 \mathrm{mag}$, where the contamination is estimated to be $\sim 35 \%$. This reduces the sample to 470 objects, which constitute our basic sample considered in the analysis below.

Our basic sample includes all cluster galaxies with $M_{B} \leq$ -16 mag classified as either E or S0, which we now want to separate into giant $\mathrm{E}$ and dwarf $\mathrm{E}^{1}$. The original method to distinguish dEs from Es is based on morphological grounds using the fact that dEs have a relatively low central surface brightness and a rather flat surface brightness profile compared to giant Es (Sandage \& Binggeli 1984; Ferguson \& Sandage 1990; Ferguson \& Binggeli 1994). On images with sufficient resolution and depth these features can be visually gauged and the classification can therefore be done by eye. As a result the transition from giants to dwarfs occurs around an absolute magnitude in the $B$-band of $M_{B} \sim-18$ mag (Sandage et al. 1985), with some overlap of the two classes. Therefore, absolute magnitudes in the range $-17<M_{B}<-19$ mag are often used to discriminate between dEs and Es (Aguerri et al. 2005; Matković \& Guzmán 2005) or even between dwarf and giant galaxies in general (Schneider et al. 1990; Patterson \& Thuan 1996; van Zee 2001; Barazza et al. 2006; Boselli et al. 2008a).

Our data do not allow to accurately separate dEs from Es visually and we have therefore to rely on another measure. As mentioned above, it is clear that applying a magnitude cut at $M_{B}=-18$ mag will not cleanly distinguish between dwarfs and giants: some giants will be in the dwarf sample and some dwarfs will be missing. This was also shown in a detailed study of the surface brightness profiles of early-type galaxies in the Virgo cluster by Kormendy et al. (2009). Dwarf and giant subsamples exhibit some overlap in absolute magnitude, but separate quite clearly with respect to central surface brightness (see Fig. 34 in Kormendy et al. 2009). This suggests that the central surface brightness is a better measure to define a dwarf sample. Figure 1 illustrates sample selections based on absolute magnitude and central surface brightness. Figure 1a shows the $M_{B}$ distribution of all early-type galaxies in A901/902 (solid+dotted histogram). The solid histogram indicates the sample considered

\footnotetext{
1 In the remainder of the paper we only use the term $\mathrm{E}$ or $\mathrm{dE}$ subsuming SOs and dSOs.
} 

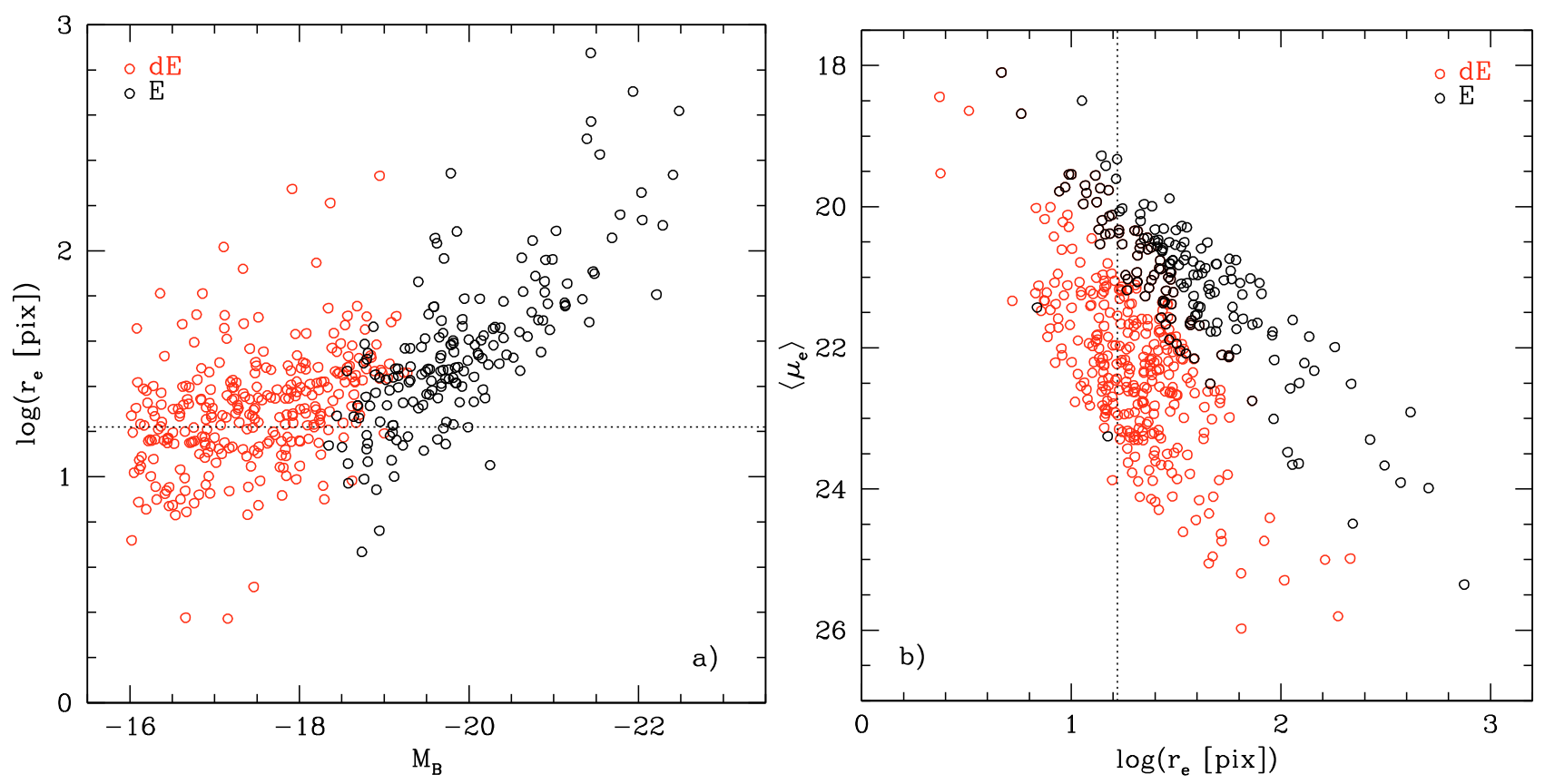

Fig. 2. Two additional photometric scaling relations for the early-type dwarf sample $\left(\mu_{0, F 606 \mathrm{~W}}>19 \mathrm{mag}\right)$ and the giant early-type sample $\mu_{0, F 606 \mathrm{~W}} \leq$ 19 mag. a) The absolute $B$-band magnitude versus the effective radius $\left(r_{\mathrm{e}}\right)$ determined by a Sérsic fit. The dotted line indicates $r_{\mathrm{e}}=1.41 \mathrm{kpc}$. b) $r_{\mathrm{e}}$ versus the mean surface brightness $\left(\left\langle\mu_{\mathrm{e}}\right\rangle\right)$ within $r_{\mathrm{e}}$. The dotted line indicates $r_{\mathrm{e}}=1.41 \mathrm{kpc}$.

in our study. A possible sample selection is indicated by the dashed line. Figure $1 \mathrm{~b}$ shows $M_{B}$ versus the central surface brightness, $\mu_{0, F 606 \mathrm{~W}}$. The latter has been determined using the central 25 pixels, corresponding to a diameter of $\sim 420 \mathrm{pc}$. The magenta triangles show the dwarf sample as defined in Fig. 1a. This definition would exclude a number of galaxies with rather low $\mu_{0, F 606 \mathrm{~W}}$. A cut applied at $\mu_{0, F 606 \mathrm{~W}}=19 \mathrm{mag} / \mathrm{arcsec}^{2}$ includes all galaxies with $M_{B}>-18$ mag and leads to two distinct sequences in a plot of effective radius versus effective surface brightness (see below, Fig. 2b). It also provides a division with smaller overlap between the samples, i.e. with less contamination, and is therefore a cleaner definition of the dwarf sample. Hence, we regard all early-type galaxies having $\mu_{0, F 606 \mathrm{~W}}>19 \mathrm{mag} / \operatorname{arcsec}^{2}$ as dEs, except seven galaxies with $\mu_{0, F 606 \mathrm{~W}}>19 \mathrm{mag} / \mathrm{arcsec}^{2}$ and $M_{B} \leq-19.5 \mathrm{mag}$, which we regard as being giant ellipticals with exceptionally low central surface brightnesses. Our sample of dEs therefore includes 295 objects.

For the sake of completeness we show in Fig. 2 two additional photometric scaling relations. In Fig. 2a we plot the absolute $B$-band magnitude versus the effective radius. The dotted line indicates an effective radius of $r_{\mathrm{e}}=1.41 \mathrm{kpc}$, which was found to be the typical size of dEs in the Virgo cluster (Boselli et al. 2008b). Slightly smaller sizes were found for dE populations in the Antlia (Smith Castelli et al. 2008) and Coma (Graham \& Guzmán 2003) clusters, however with significantly smaller samples sizes. Figure 2a shows that among Es brighter galaxies have large effective radii. For dEs the scatter is much larger and a similar sequence cannot be clearly identified. This is primarily caused by the rather small magnitude range covered by our dE sample (for a comparison see, e.g., de Rijcke et al. 2009, Fig. 2). In Fig. $2 b$ we plot the effective radius versus the mean surface brightness within $r_{\mathrm{e}}$. Giants and dwarfs form two separated sequences of decreasing surface brightness with increasing effective radius. The sequence for Es is a possible projection of the fundamental plane of elliptical galaxies and the fact that we obtain two distinct sequences in this plot supports our definition

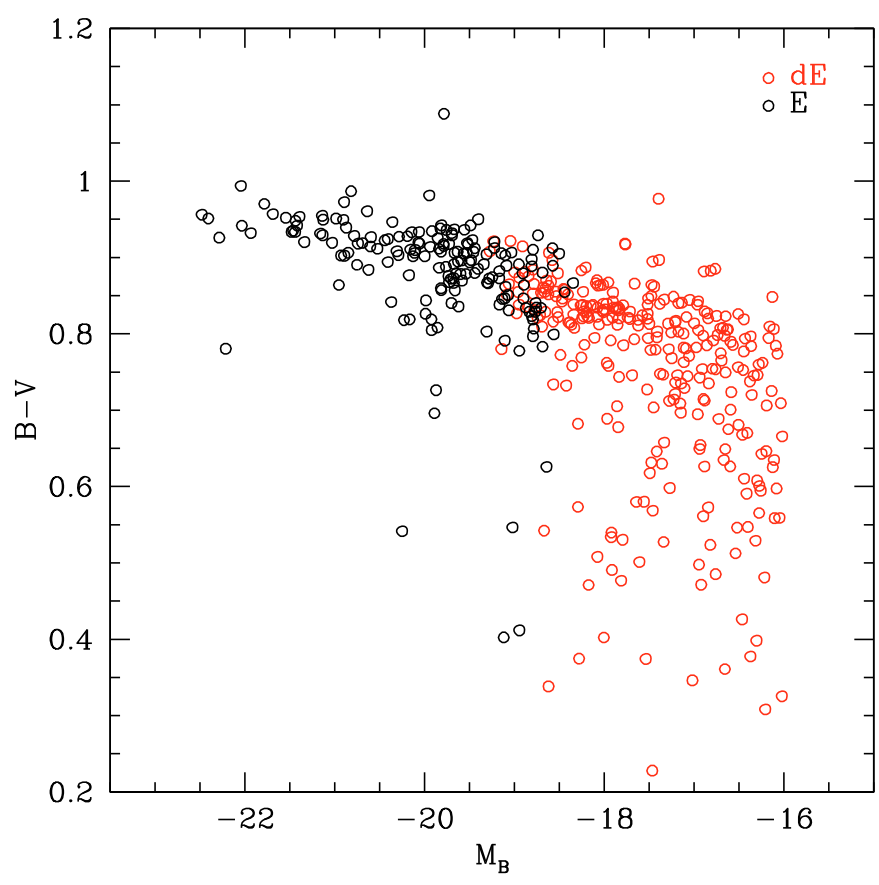

Fig. 3. The color-magnitude diagram for our sample of early-type galaxies. The dwarf and giant subsamples are defined as discussed in Sect. 2.3. The typical error of the $B-V$ color is in the range $0.10-0.15$ mag.

of dwarfs and giants. However, the $\mathrm{dE}$ sequence is also caused by the fact that we exclude galaxies with $M_{B}>-16$ mag, which would otherwise appear in the lower left corner of the plot.

\section{The color-magnitude relation}

Early-type galaxies are typically forming the red sequence in a plot of absolute magnitude versus color (the color-magnitude 
diagram, CMD). In Fig. 3 we show such a plot for our earlytype galaxy sample. In fact, Es and a majority of dEs form a rather tight relation in this plot. However, there are a number of dEs, which are significantly bluer than expected based on their magnitude. We emphasize that these objects have been classified as early-type galaxies, thus they appear to be blue dEs. They are therefore different than the dEs with blue central colors observed in local dwarf galaxy samples (Lisker et al. 2006; Tully $\&$ Trentham 2008). We have reinspected all galaxies, with large separations from the red sequence and found that their morphological classification is in general correct. This is illustrated in Fig. 4. The top three rows show dEs on the red sequence. Each galaxy is represented by a $F 606 W$-band image (first column), a color representation of the HST images based on $B V R$ colors from COMBO-17 (second column), and by the residual after subtracting a Sérsic model from the $F 606 \mathrm{~W}$-band image (third column). The bottom three rows show the same for dEs with significantly bluer colors than expected based on their absolute magnitude. The visual classification was performed using the $F 606 \mathrm{~W}$-band images. In particular the $B V R$-based representations demonstrate the different colors.

A possible reason for the blue colors of these objects could be a wrong redshift. The contamination of our $\mathrm{dE}$ sample by field galaxies is on the order of $<10 \%$ down to $M_{B} \sim-19$ mag and $\sim 35 \%$ at $M_{B}=-16 \mathrm{mag}$ (Gray et al. 2009). The possible contamination therefore roughly corresponds to the fraction of galaxies below the red sequence. For instance, a disk galaxy effectively located at a higher redshift could appear very similar to a closer $\mathrm{dE}$, while maintaining its blue color. This interpretation is supported by the residual images shown in the third column of Fig. 4. These residuals were obtained by subtracting the best fitting Sérsic model from the $F 606 \mathrm{~W}$-band image. While the red $\mathrm{dEs}$ hardly show any residual (top three rows), the blue dEs exhibit strong residuals resembling rings, bars, or even spiral arms (bottom three rows). We emphasize that such strong residuals are found in a large majority of blue dEs.

These rather blue dEs with large separations from the red sequence are either very interesting objects in terms of possible formation scenarios of dEs or they are simply contaminants, which effectively are at higher redshifts. We cannot distinguish between these two possibilities on an individual basis, but we assume that a majority are not cluster members. We will obtain a better assessment of their cluster membership through the analysis of low resolution prism spectroscopy with Magellan (IMACS/PRIMUS) for galaxies down to $R=23.6 \mathrm{mag}$. These observations have already been performed and the data analysis is underway. For now we include these galaxies in the following analysis and show them as a separate subsample as defined below.

Figure 3 has shown that Es and a majority of dEs form a red sequence in a CMD. Now we want to compare this red sequence to the one found for dEs in the Virgo cluster and use the corresponding fit to further subdivide our dE sample. In Fig. 5 we plot a CMD in SDSS $u$ - and $r$-bands (we use the SDSS filters here, since the Virgo study has been performed with SDSS data). The solid line is the fit to the red sequence of dEs in the Virgo cluster obtained in the study of Lisker et al. (2008). We use this fit rather than a fit to our own data, because it is based on a dE sample covering a much larger range in magnitudes. The dEs in our sample are in good agreement with the Virgo fit, while the majority of Es are shifted towards redder colors. A nonlinear color-magnitude relation for a combined sample of giant and dwarf early-type galaxies has been reported by Janz \& Lisker (2009), while linear color-magnitude relations have been found in recent photometric studies of nearby galaxy clusters (Misgeld et al. 2008, 2009; Smith Castelli et al. 2008). We use this fit to further subdivide our dE sample, which is indicated in Fig. 5 by the red, green, and blue circles. We define three subsamples based on the fit and on a parallel shift of the fit by 0.25 mag to the blue (dotted line): dEs with redder colors than expected based on their magnitude ("red dEs", 103 objects), the subsample of dEs with bluer colors ("intermediate dEs", 126 objects), and the subsample of dEs, which exhibit rather large separation from the red sequence as discussed above ("blue dEs", 66 objects).

This approach is based on the assumption that the interaction of the galaxies with the cluster causes them to move from the blue cloud to the red sequence on the CMD. This process takes some time and the change in color of a galaxy is not occurring at once. In this picture, the redder dEs are subjected to this process for a longer time than the bluer dEs and are also believed to have an older stellar population (see the discussion of corresponding results in Sect. 4). By separating the dEs along the red sequence fit, we are trying to investigate this process in the following.

\section{The distribution of dEs in A901/902}

The distribution of early-type galaxies in the multiple-cluster system is shown in Fig. 6. Four distinct subcomponents have been identified in A901/902 (A901a, A901b, A902, and the South West group (SW group), Gray et al. 2002; Heymans et al. 2008), which are indicated by black crosses in Fig. 6a. The circles have a radius of $\sim 500 \mathrm{kpc}$. The galaxies are clearly concentrated towards the four structure centers. In particular the red $\mathrm{dEs}$ avoid the region between the subcomponents (central region in Fig. 6a). In a next step we determine the projected distances (in arcsec) of all galaxies to the nearest subcomponent. We bin these distances in four distance ranges and show the corresponding fraction of each subsample within these bins in Fig. 6b. Also shown is the result for a random distribution of galaxies. It is based on the average of 1000 random distributions of 180 galaxies in the cluster system. As indicated by Fig. 6a, the red dEs are strongly concentrated towards the centers of the subclumps, with a fraction of $\sim 56 \%$ within the first two bins (corresponding to $\sim 560 \mathrm{kpc}$ ). Only $\sim 35 \%$ of the intermediate dE population is located within the same radius. A KS-test shows that the probability that the red and intermediate subsamples stem from the same parent distribution is $<0.5 \%$. The distribution for Es is rather flat, i.e., still strongly concentrated towards the centers as opposed to a random distribution (magenta line), but to a lesser degree than red dEs. The population of blue dEs shows no concentration towards the centers and their distribution is the same (within errors) as the one expected for a unrelated field population (magenta line).

The difference between the distributions of red and intermediate dEs corresponds to a color-density or color-radius relation, after the correlation between color and luminosity has been removed. Hence, the scatter of the red sequence for dEs in Fig. 5 is related to the distribution of the galaxies in the cluster region. Figure 7 shows the $U-V$-color as a function of distance to the nearest cluster center for red and intermediate dEs. The overall color-density relation of the dEs on the red sequence is in fact quite weak, showing the importance of the normalization by luminosity.

The fact that early-type dwarfs are strongly concentrated towards the centers of their host clusters was also found in studies of local galaxy clusters (Binggeli et al. 1987; Ferguson \& Sandage 1989; Lisker et al. 2007). In particular, nucleated dEs exhibit a stronger concentration towards the cluster center than 

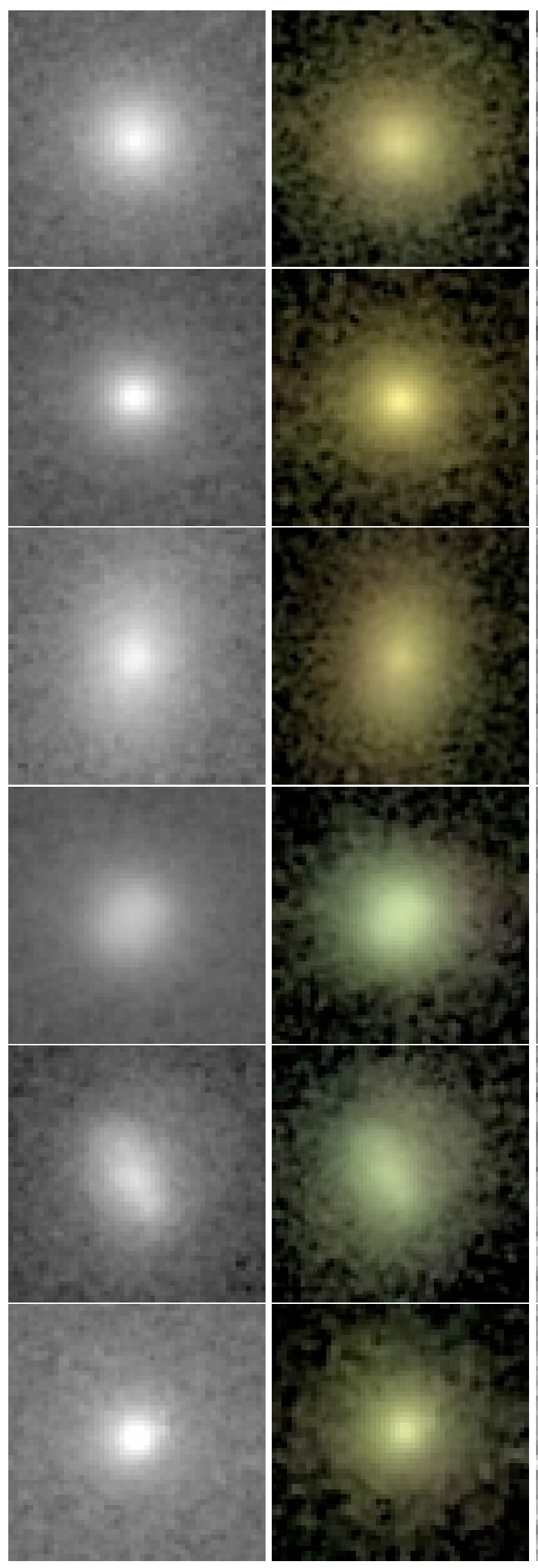

$M_{B}=-17.79 \mathrm{mag}$

$\log \left(r_{e}[\right.$ pix $\left.]\right)=1.16$

$\left\langle\mu_{e}\right\rangle=21.39 \mathrm{mag}$

$M_{B}=-17.51 \mathrm{mag}$

$\log \left(r_{e}[\right.$ pix $\left.]\right)=1.10$

$\left\langle\mu_{e}\right\rangle=21.23 \mathrm{mag}$

$M_{B}=-17.83 \mathrm{mag}$ $\log \left(r_{e}[\right.$ pix $\left.]\right)=1.35$

$\left\langle\mu_{e}\right\rangle=22.33 \mathrm{mag}$

$M_{B}=-17.79 \mathrm{mag}$

$\log \left(r_{e}[\right.$ pix $\left.]\right)=0.92$

$\left\langle\mu_{e}\right\rangle=20.42 \mathrm{mag}$

Fig. 4. Examples of dEs on the red sequence and dEs with large separations from the red sequence. First column: the $F 606 W$-band stamp; second column: a color representation of the HST images based on $B V R$ colors from COMBO-17 of the same galaxies; third column: residual after subtracting a Sérsic model of the same galaxies; fourth column: photometric parameters as plotted in Fig. 2. The top three rows show dEs on the red sequence and the bottom three rows show dEs with large separations from the red sequence. All images are $\sim 4.2 \mathrm{kpc}$ on a side. 


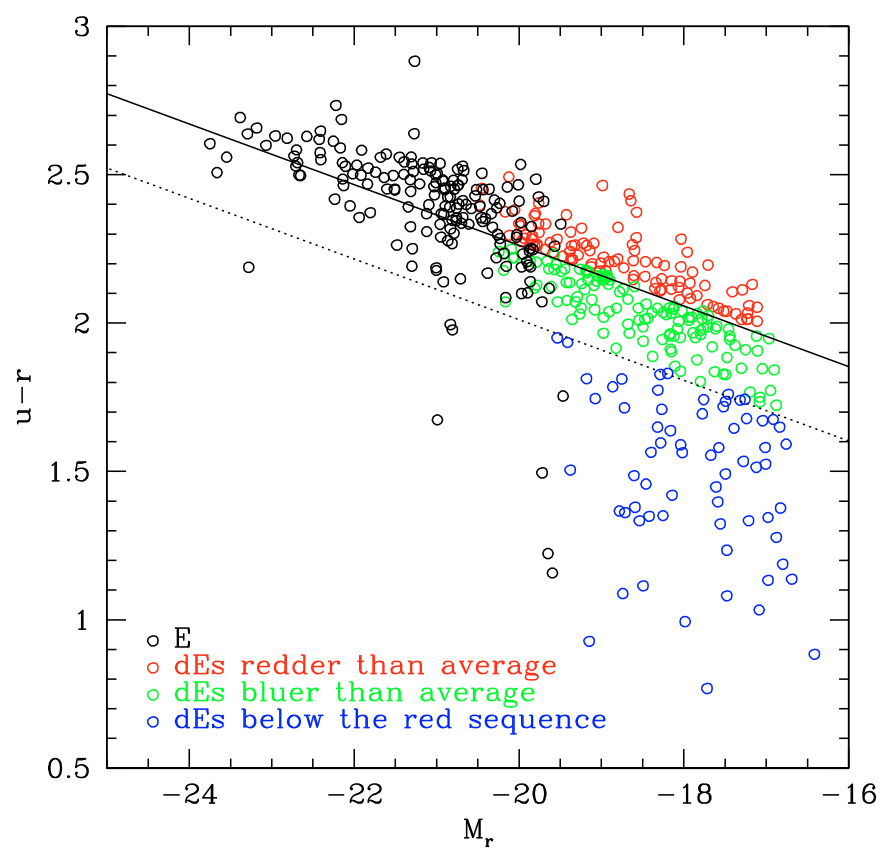

Fig. 5. The CMD in SDSS $u$ - and $r$-bands. The solid line is a fit to the red sequence of dEs in the Virgo cluster from Lisker et al. (2008). The dotted line is the same, but shifted by 0.25 mag to the blue. These lines are used to define three subsamples of dEs. The color has a typical error in the range $0.10-0.15 \mathrm{mag}$.

the typically fainter non-nucleated dEs. A finding, we cannot verify with our data (see below). A significant trend of dE color with clustercentric radius in the Coma cluster, where redder dEs tend to be closer to the cluster center, was reported by Secker (1996); although the scatter of the data points was rather large. A similar result based on the color-magnitude relations for different dEs subpopulations was also found by Lisker et al. (2008) for the Virgo cluster. Moreover, Wolf et al. (2007) find evidence for a relation between the residuals from the color-magnitude relation and local galaxy density for objects in the range $-18<$ $M_{V}<-20$ mag using the COMBO-17 data for A901/902. On the other hand, Sánchez-Janssen et al. (2008) did not find a color gradient among red dwarf galaxies in a large compilation of galaxy clusters mapped by the SDSS. In general the gradients found are weak and only significant taking into account projection effects and assuming that these will always weaken an intrinsic color gradient. Finally, age gradients in the sense that older dEs are closer to the cluster centers were found in the Virgo (Michielsen et al. 2008) and Coma clusters (Smith et al. 2009). These studies also show that the dEs lying closer to the cluster centers tend to be more metal poor. Together with the results presented in our study, this indicates that the color differences among dEs with the same luminosity are more related to their ages than to their metallicities.

\section{The compactness of dEs}

Many dEs are found to harbor nuclei, which most likely are massive compact star clusters like the nuclei in nearby lowluminosity spirals (Kormendy \& McClure 1993; Phillips et al. 1996; Matthews et al. 1999; Böker et al. 2002). The nuclei in dEs are located at or near the center of the luminosity distribution of the host galaxy (Binggeli et al. 2000; Barazza et al. 2003) and have sizes in the range $\lesssim 2 \sim 60$ pc (Geha et al. 2002; Côté et al. 2006). In models of $\mathrm{dE}$ formation, nucleated dEs (or dENs) are predicted to be more strongly concentrated towards the cluster centers than non-nucleated dEs, since the former are more robust against the impact of the environment (Moore et al. 1998). This property of the $\mathrm{dEN}$ population was reportedly found in the Virgo cluster (van den Bergh 1986; Binggeli et al. 1987; Lisker et al. 2007), but the result is still controversial (Côté et al. 2006).

Due to their small sizes, the nuclei in our dEs cannot be resolved and their identification is therefore very uncertain. However, in order to investigate, whether the more robust dEs exhibit a stronger clustercentric concentration, we can rely on other measures of compactness, indicating the possible presence of a nucleus. We have shown in Fig. $6 \mathrm{~b}$ that the red dEs are more centrally concentrated within the cluster than the intermediate dEs. Now we test, whether the former are more compact than the latter. In Fig. 8 we show the histograms of different compactness indicators for our subsamples. Figure $8 \mathrm{a}$ shows the distributions of the central surface brightness for red and intermediate dEs. The two distributions are indeed different: red dEs have higher central surface brightnesses with a median of $\left\langle\mu_{0, F 606 \mathrm{~W}}\right\rangle=$ $20.27 \mathrm{mag} / \mathrm{arcsec}^{2}$ compared to $\left\langle\mu_{0, F 606 \mathrm{~W}}\right\rangle=20.86 \mathrm{mag} / \mathrm{arcsec}^{2}$ for intermediate dEs. A KS-test shows that the probability that the two samples are drawn from the same parent distribution is $<1 \%$. For the Sérsic index $n$, shown in Fig. $8 \mathrm{~b}$, we obtain a similar results: the median values are $\langle n\rangle=2.45$ and $\langle n\rangle=2.12$ for red and intermediate $\mathrm{dEs}$, respectively. The KS probability is $<0.1 \%$. The result is less pronounced for the concentration index $C$ (Fig. 8c), derived using the CAS code (Conselice et al. 2000; Heiderman et al. 2009). For $C$ we find $\langle C\rangle=3.61$ for red dEs and $\langle C\rangle=3.55$ for intermediate dEs, with a KS probability of $\sim 3 \%$. These findings indicate that dEs located close to the cluster centers are on average redder and more compact than their counterparts at larger radii. Moreover, the results also support the expectation that nucleated dEs should be more centrally concentrated within the cluster than non-nucleated dEs. This view is also based on the fact that our two dE subsamples show significant differences in terms of $\mu_{0, F 606 \mathrm{~W}}$ and $n$, which are better tracers of the presence of a nucleus than $C$, since they are more sensitive to the very central part of the surface brightness profiles of the objects. Finally, Fig. 8d shows the distributions of the axis ratios $(q)$. Red dEs tend to be rounder $(\langle q\rangle=0.72)$ than intermediate dEs $(\langle q\rangle=0.64$, KS probability $\sim 7 \%)$. In fact, $\mathrm{dENs}$ are found to be rounder on average than non-nucleated dEs (Binggeli \& Popescu 1995), which provides further support for our finding that more compact (and possibly nucleated) dEs exhibit a stronger concentration towards the cluster centers than the $\mathrm{dE}$ population in general.

\section{Discussion}

The main finding of our study is a color-density relation among dEs in the sense that for galaxies with the same luminosity the redder objects are located closer to the cluster center than the bluer ones. In addition, the redder dEs tend to be more compact and rounder. These findings indicate that fundamental properties of dEs are affected by the interaction with the cluster environment. In particular the star formation history (color-density relation) and the structure (compactness and roundness) of dEs seem to depend on their specific location within the cluster region.

Before discussing possible processes that could have led to our findings, we have to point out that the dE population in clusters covers a wide range of luminosities, reaching magnitudes as faint as $M_{B} \sim-10$ mag (Sabatini et al. 2003). It is therefore likely that different formation processes are at work and that the faint dEs have a different origin than their brighter 

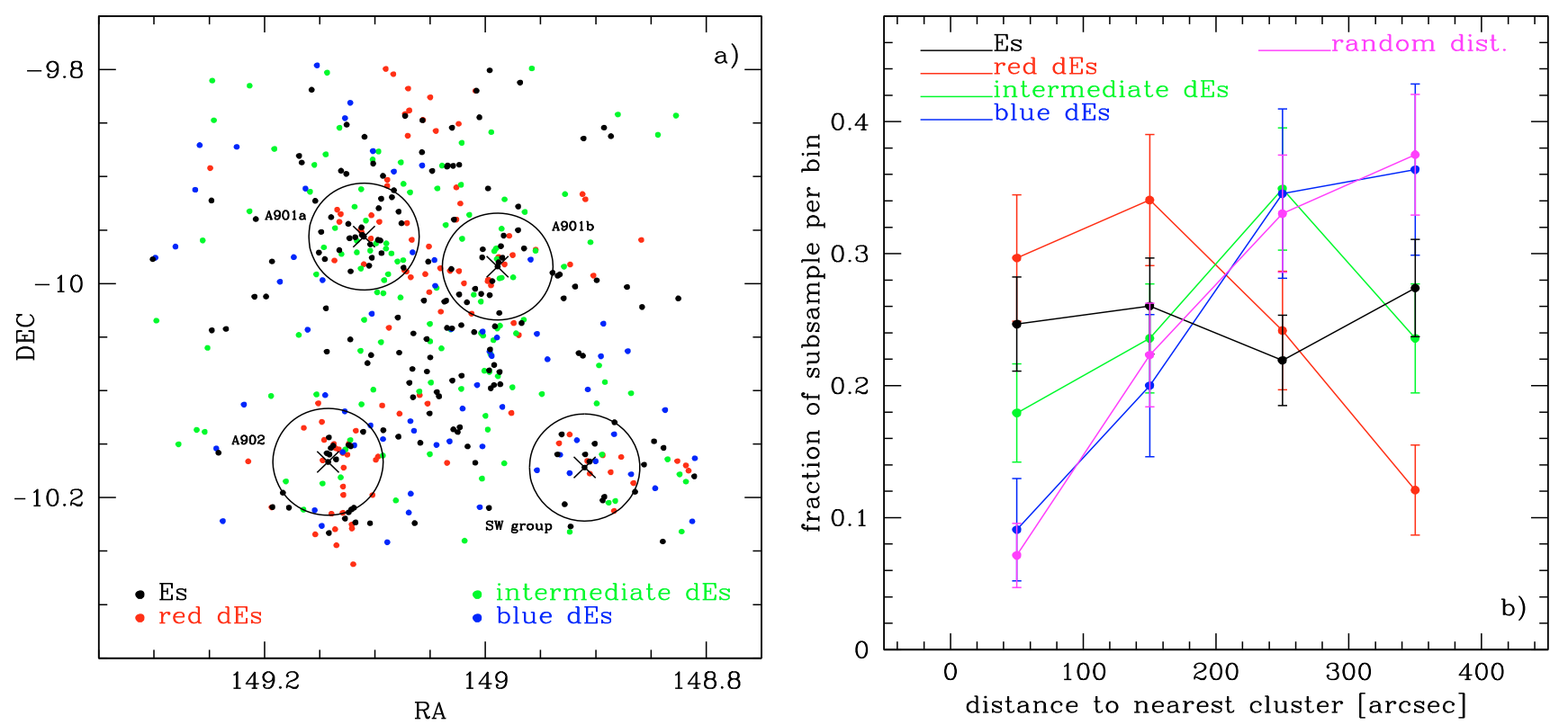

Fig. 6. a) A map of the multiple-cluster system A901/902 showing the distribution of all early-type galaxies. The different colors represent the subsamples as defined in Fig. 5. The circles have a radius of $\sim 500 \mathrm{kpc}$. The centers of the four subcomponents of the multiple-cluster system are indicated by black crosses. b) The fraction of each subsample and a random distribution of galaxies in four distance (to the nearest cluster) bins.

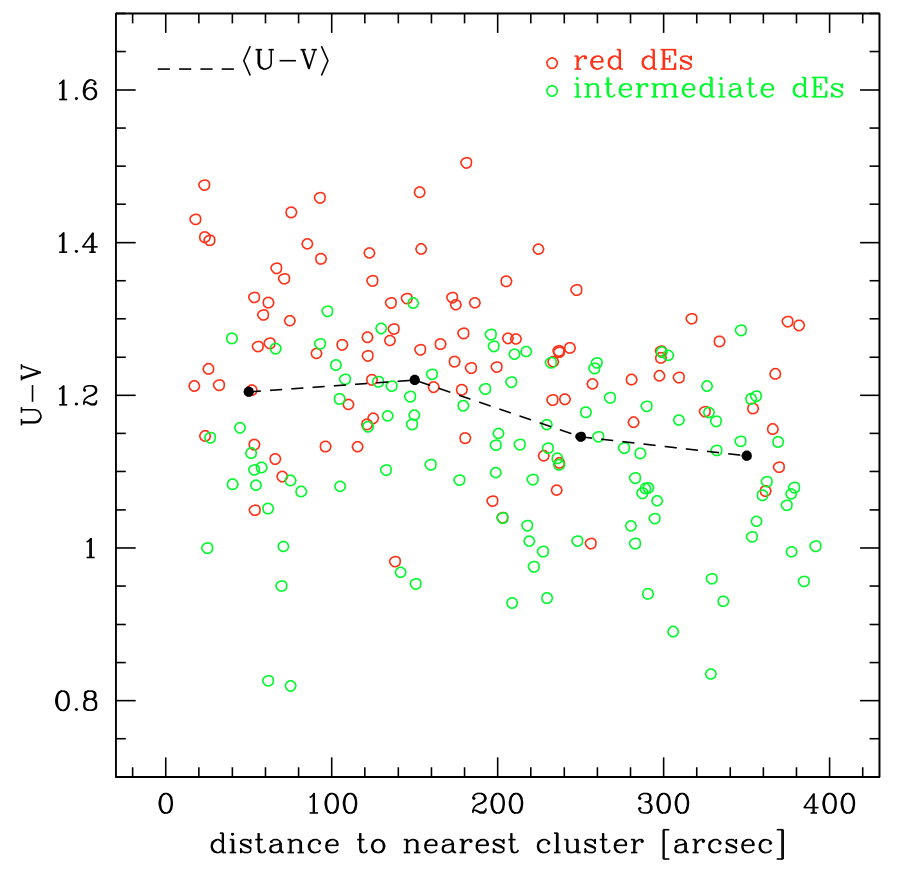

Fig. 7. The total $U-V$ color of red and intermediate dEs as a function of distance to the nearest cluster center. The filled circles show the average colors in four distance bins.

counterparts. Since our sample is dominated by rather luminous objects $\left(M_{B} \leq-16 \mathrm{mag}\right)$, our discussion concentrates on the possible processes leading to the formation of bright dEs.

\subsection{Ram pressure stripping}

Ram pressure stripping describes the effect of the inter-cluster medium (ICM) on the inter-stellar material (ISM) of a galaxy moving through the cluster (Gunn \& Gott 1972). If the density of the ICM is high enough, its pressure can lead to the total or partial removal of the ISM from the moving galaxy. Ram pressure stripping is more efficient in lower mass galaxies and has therefore been invoked as a possible process that terminates star formation in dwarf irregulars and turns them into quiescent dwarf ellipticals in clusters (Lin \& Faber 1983; Abadi et al. 1999; van Zee et al. 2004). Observational evidence for ram pressure stripped galaxies in nearby clusters has been presented in recent studies (e.g., Vollmer et al. 2004; Kantharia et al. 2008). Since the density of the ICM is increasing towards the cluster centers, it is plausible that galaxies with smaller clustercentric distances experience a stronger impact from ram pressure stripping than galaxies at larger radii. This can affect their star formation histories. The ISM of galaxies close to the cluster centers might be removed faster and more efficiently, and as a consequence their star formation terminates earlier compared to their counterparts at larger radii. The former would then be dominated by an older stellar population than the latter. Thus, our finding of a colordensity relation is consistent with this picture. The fact that we find a stronger relation using color residuals, i.e. taking into account the luminosity of the galaxies, could be due to the fact that the strength of ram pressure stripping also depends on the mass of the affected galaxy. On the other hand, our additional results that the compactness and axis ratios of dEs increase towards the cluster centers are more difficult to reconcile with this scenario. It is not clear how the mere removal of gas can cause these structural signatures. For a discussion of possible explanations and related issues see Boselli et al. (2008a).

\subsection{Galaxy harassment}

Galaxy harassment describes the total morphological transformation of disk galaxies into quiescent spheroidals in a galaxy cluster through repeated encounters with more massive galaxies and the cluster's tidal field (Moore et al. 1998). Hence, this process is believed to be able to transform late-type disk galaxies into dEs. Further $N$-body simulations have shown (Mastropietro et al. 2005) that in some cases the transformation remnants can maintain parts of their original disk structure within a spheroidal component. Such hidden disk structures have been found in a 

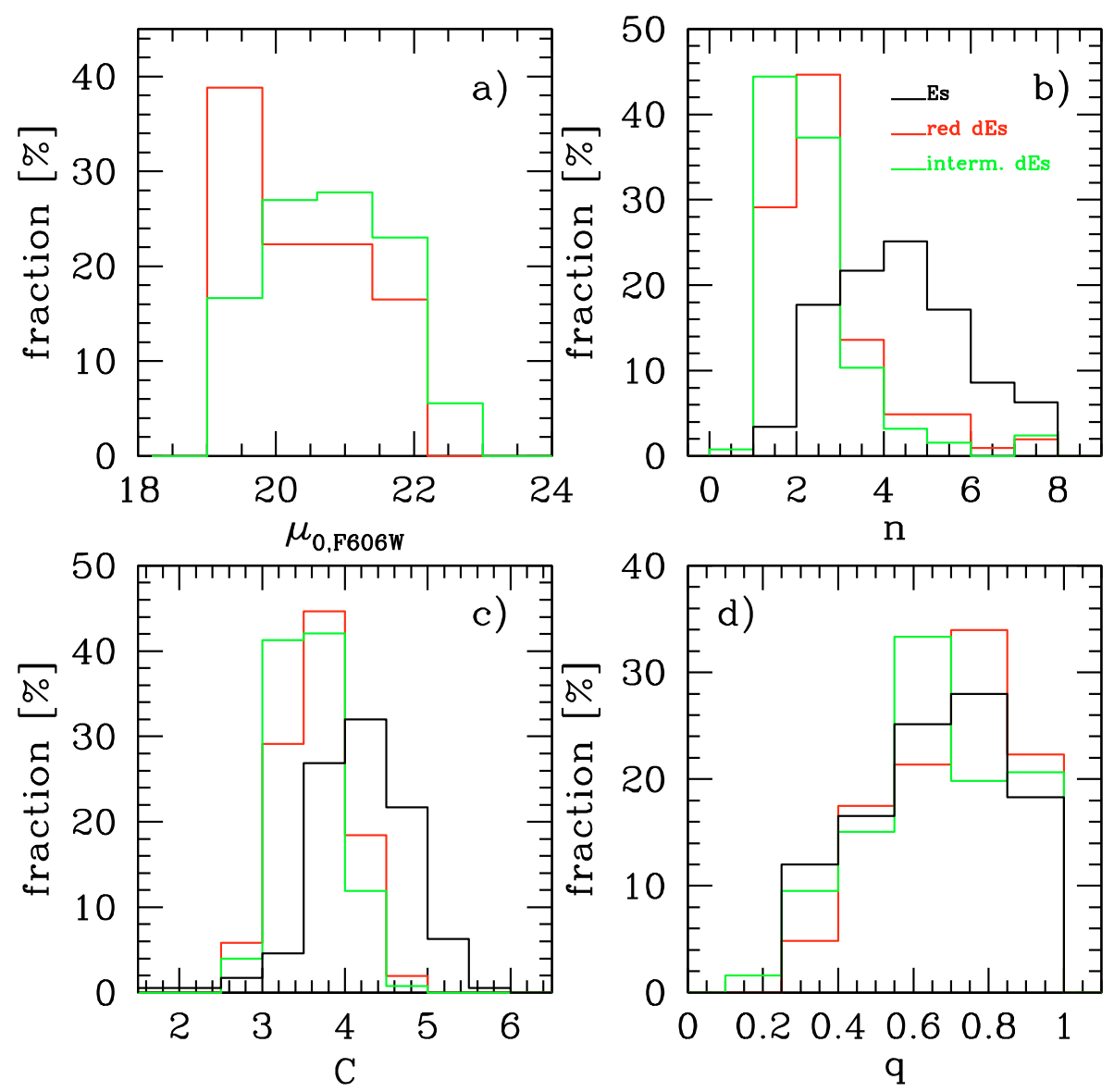

Fig. 8. The distributions of different measures of compactness and roundness for red dEs (red histograms), intermediate dEs (green histograms), and giant Es (panels b)-d), black histograms). a) Central surface brightness, $\mu_{0, F 606 \mathrm{~W}}$, as defined in Sect. 2.3; b) Sérsic in$\operatorname{dex} n ; \mathbf{c})$ concentration index $C ; \mathbf{d})$ axis ratio $q$, defined as semi-major axis divided by semiminor axis.

number of bright dEs in different nearby clusters where spiral or bar features have been observed (Jerjen et al. 2000; Barazza et al. 2002; Graham et al. 2003; De Rijcke et al. 2003; Lisker et al. 2006). The model can also account for the presence of compact nuclei in the centers of many cluster dEs (Sandage \& Binggeli 1984; Ferguson \& Binggeli 1994), which have also been observed in a large fraction of nearby late-type disk galaxies (Carollo et al. 1997; Böker et al. 2002). Moreover, the galaxy harassment model predicts that the surviving ensemble of dEs exhibit a color- or age- (also metallicity) density relation within the galaxy cluster and that the fraction of nucleated dEs increases towards the cluster center (Moore et al. 1998). The colordensity relation found for the A901/902 dEs is therefore consistent with the harassment scenario. Our finding that the redder $\mathrm{dEs}$ are on average more compact and rounder than bluer dEs also agrees with the model predictions, since nucleated dEs have been found to be rounder than non-nucleated dEs (Binggeli \& Popescu 1995). Therefore the model predicts an ellipticity gradient among dEs, which can be inferred from our results. On the other hand, we do not find a gradient in terms of effective radius, which is also predicited by the harassment model (Mastropietro et al. 2005). The remnants of the interactions in the outskirts of the cluster are found to have larger effective radii by a factor of $\sim 2$ compared to the ones at smaller clustercentric distances. We do not find a corresponding difference between red and intermediate dEs, which have roughly the same average effective radii. Finally, we want to point out that support for the assumption that late-type, intermediate-mass disk galaxies are affected by the cluster is provided by a study of the environmental dependence of the stellar mass-size relation in A901/902 using STAGES data (Maltby et al. 2009). The results indicate that almost all galaxies show no difference in the stellar mass-size relation between cluster and field. However, the only galaxies to show a possible difference were the class of intermediate-mass $\left(\log M<10 M_{\odot}\right)$ spirals, where the spirals in the cluster were found to have a smaller mean effective radius than their counterparts in the field at the $2 \sigma$ level.

\subsection{Preprocessing in galaxy groups}

Galaxies are not necessarily joining a cluster as individuals, but as part of a galaxy group. Hence, part of their evolution might take place in the group environment prior to their accretion to a galaxy cluster. Therefore, these galaxies were preprocessed before they experienced the impact of the interaction with the cluster environment (Cortese et al. 2006; Kautsch et al. 2008; Li et al. 2009). It has been shown by Mayer et al. (2001b) that star forming dwarf galaxies can be transformed into early-type dwarfs in a Milky Way sized halo by tidal stirring. These models also predict relations between galaxy properties and their distance to the parent galaxy (Mayer et al. 2001a). The Abell 901/902 cluster system is composed of four distinct subcomponents, whose centers are indicated in Fig. 6a and were used to define the radial distributions in Fig. 6b. However, only the SW group can be regarded as a typical galaxy group, the other substructures are more massive. Their central velocity dispersions are of the order of $\sigma \sim 600-900 \mathrm{~km} \mathrm{~s}^{-1}$ (Heiderman et al. 2009), which place them between the Virgo cluster $\left(\sigma \sim 400-750 \mathrm{~km} \mathrm{~s}^{-1}\right.$, Binggeli et al. 1987) and the Coma cluster $\left(\sigma \sim 900 \mathrm{~km} \mathrm{~s}^{-1}\right.$, The \& White 1986). The SW group is smaller $\left(\sigma \sim 500 \mathrm{~km} \mathrm{~s}^{-} 1\right)$ and contributes $13(\sim 13 \%)$ and 18 objects $(\sim 14 \%)$ to our red and intermediate subsamples, respectively. Their distribution with respect to the center of the SW group is very similar to the one shown 
for the entire subsamples in Fig. 6b. The SW group might therefore represent an example of a (rather massive) galaxy group delivering preprocessed dwarf galaxies to a larger galaxy system. However, we want to point out that the remnants in simulations of group related processes are commonly rather small, low mass dwarfs comparable to the dwarf spheroidal galaxies in the Local Group and therefore very different from the bright dEs in our sample.

The fact that dEs are very abundant in galaxy clusters, which can strongly affect their members, does not necessarily prove that they are the results of transformation processes. It is quite possible that some dEs belong to the primordial cluster population and experienced the impact from the cluster environment throughout their evolution leading to the objects we observe today. On the other hand, it is clear that galaxies can be profoundly affected by the interactions with a galaxy cluster. The agreement between our findings and the predictions of the cluster related processes discussed above is consistent with a scenario in which a considerable fraction of dEs in A901/902 have been affected by their environment.

\section{Summary and conclusions}

We have used data from the multiwavelength survey STAGES (Space Telescope A901/902 Galaxy Evolution Survey, Gray et al. 2009) to study the population of bright dEs in the multiplecluster system Abell A901/902. Our definition of dEs is based on the identification of early-type galaxies through visual classifications and a separation of dwarfs and giants using a cut in central surface brightness at $\mu_{0, F 606 \mathrm{~W}}=19 \mathrm{mag}$. We subdivide our $\mathrm{dE}$ sample into red, intermediate, and blue dEs according to their position on a CMD using $M_{r}$ and $u-r$. The corresponding $\mathrm{dE}$ subsamples exhibit very different distributions within the cluster region with respect to the distance to the nearest cluster center. The red dEs are strongly concentrated towards the cluster centers, even stronger than giant Es, while the distribution of the intermediate dEs is comparable to the one of Es and the blue dEs avoid the cluster centers. The distribution of the latter sample is similar to the one of a field population and we cannot rule out that a large fraction of objects in this sample are contaminants seen in projection within the cluster region. The different distributions of red and intermediate dEs is interpreted as a color-density relation with respect to the cluster centers in the sense that for dEs with similar luminosities the redder galaxies are more centrally concentrated in the cluster than the bluer objects. Furthermore, we find evidence that the red dEs are more compact, indicating the presence of a nucleus, and rounder than the bluer subsample of dEs.

The relation between fundamental properties of dEs and their location with respect to the cluster centers suggests that the formation of dEs is strongly affected by cluster related processes. We therefore compare our results to the model predictions based on processes that are believed to take place in a cluster environment. Our findings are consistent with a number of these predictions and indicate that a considerable fraction of dEs in A901/902 are the result of transformations. It is plausible that several processes are involved and affect the evolution of the cluster galaxies to some degree. However, our data are not sufficient to determine the contributions of the different factors shaping the $\mathrm{dE}$ population in A901/902 and to provide evidence that transformations are involved at all. On the other hand, we find good agreement between our findings and the predictions of the galaxy harassment model indicating that processes described in this scenario are most likely contributing to the formation and evolution of at least some dEs in A901/902.

Acknowledgements. We thank the anonymous referee for a helpful and constructive report. C.W. and M.E.G. were supported by an STFC Advanced Fellowship. S.J., A.H., and I.M. gratefully acknowledge support from NSF grant AST0607748, LTSA grant NAG5-13063, as well as programs HST GO-10395, HST GO-10861, and HST GO-11082, which were supported by NASA through a grant from the Space Telescope Science Institute, which is operated by the Association of Universities for Research in Astronomy, Incorporated, under NASA contract NAS5-26555. M.B. was supported by FWF grant P18416. Support for STAGES was provided by NASA through GO-10395 from STScI operated by AURA under NAS5-26555.

\section{References}

Abadi, M. G., Moore, B., \& Bower, R. G. 1999, MNRAS, 308, 947

Aguerri, J. A. L., Iglesias-Páramo, J., Vílchez, J. M., Muñoz-Tuñón, C., \& Sánchez-Janssen, R. 2005, AJ, 130, 475

Babul, A., \& Rees, M. J. 1992, MNRAS, 255, 346

Barazza, F. D., Binggeli, B., \& Jerjen, H. 2002, A\&A, 391, 823 Barazza, F. D., Binggeli, B., \& Jerjen, H. 2003, A\&A, 407, 121 Barazza, F. D., Jogee, S., Rix, H.-W., et al. 2006, ApJ, 643, 162 Beasley, M. A., Strader, J., Brodie, J. P., Cenarro, A. J., \& Geha, M. 2006, AJ, 131,814

Beasley, M. A., Cenarro, A. J., Strader, J., \& Brodie, J. 2009, AJ, 137, 5146

Bell, E. F., Papovich, C., Wolf, C., et al. 2005, ApJ, 625, 23

Bershady, M. A., Jangren, A., \& Conselice, C. J. 2000, AJ, 119, 2645

Binggeli, B., \& Cameron, L. M. 1991, A\&A, 252, 27

Binggeli, B., \& Popescu, C. C. 1995, A\&A, 298, 63

Binggeli, B., Sandage, A., \& Tammann, G. A. 1985, AJ, 90, 1681

Binggeli, B., Tammann, G. A., \& Sandage, A. 1987, AJ, 94, 251

Binggeli, B., Tarenghi, M., \& Sandage, A. 1990, A\&A, 228, 42

Binggeli, B., Barazza, F., \& Jerjen, H. 2000, A\&A, 359, 447

Böker, T., Laine, S., van der Marel, R. P., et al. 2002, AJ, 123, 1389

Borch, A., Meisenheimer, K., Bell, E. F., et al. 2006, A\&A, 453, 869

Boselli, A., \& Gavazzi, G. 2006, PASP, 118, 517

Boselli, A., Boissier, S., Cortese, L., \& Gavazzi, G. 2008a, ApJ, 674, 742

Boselli, A., Boissier, S., Cortese, L., \& Gavazzi, G. 2008b, A\&A, 489, 1015

Caldwell, N. 1983, AJ, 88, 804

Carollo, C. M., Stiavelli, M., de Zeeuw, P. T., \& Mack, J. 1997, AJ, 114, 2366

Conselice, C. J., Bershady, M. A., \& Jangren, A. 2000, ApJ, 529, 886

Conselice, C. J., Bershady, M. A., Dickinson, M., \& Papovich, C. 2003, AJ, 126, 1183

Cortese, L., Gavazzi, G., Boselli, A., et al. 2006, A\&A, 453, 847

Côté, P., Piatek, S., Ferrarese, L., et al. 2006, ApJS, 165, 57

De Rijcke, S., Dejonghe, H., Zeilinger, W. W., \& Hau, G. K. T. 2001, ApJ, 559, L21

De Rijcke, S., Dejonghe, H., Zeilinger, W. W., \& Hau, G. K. T. 2003, A\&A, 400, 119

de Rijcke, S., Penny, S. J., Conselice, C. J., Valcke, S., \& Held, E. V. 2009, MNRAS, 393, 798

Ferguson, H. C., \& Binggeli, B. 1994, A\&ARv, 6, 67

Ferguson, H. C., \& Sandage, A. 1989, ApJ, 346, L53

Ferguson, H. C., \& Sandage, A. 1990, AJ, 100, 1

Ferrarese, L., Côté, P., Jordán, A., et al. 2006, ApJS, 164, 334

Gavazzi, G., Donati, A., Cucciati, O., et al. 2005, A\&A, 430, 411

Geha, M., Guhathakurta, P., \& van der Marel, R. P. 2002, AJ, 124, 3073

Geha, M., Guhathakurta, P., \& van der Marel, R. P. 2003, AJ, 126, 1794

Graham, A. W., \& Guzmán, R. 2003, AJ, 125, 2936

Graham, A. W., Jerjen, H., \& Guzmán, R. 2003, AJ, 126, 1787

Gray, M. E., Taylor, A. N., Meisenheimer, K., et al. 2002, ApJ, 568, 141

Gray, M. E., Wolf, C., Barden, M., et al. 2009, MNRAS, 393, 1275

Gunn, J. E., \& Gott, J. R. I. 1972, ApJ, 176, 1

Haines, C. P., Gargiulo, A., La Barbera, F., et al. 2007, MNRAS, 381, 7

Heiderman, A., Jogee, S., Marinova, I., \& the STAGES collaboration 2009, ApJ, 705,1433

Heymans, C., Gray, M. E., Peng, C. Y., et al. 2008, MNRAS, 385, 1431

Janz, J., \& Lisker, T. 2009, ApJ, 696, L102

Jerjen, H., \& Binggeli, B. 1997, The Nature of Elliptical Galaxies; 2nd Stromlo Symposium, 116, 239

Jerjen, H., Kalnajs, A., \& Binggeli, B. 2000, A\&A, 358, 845

Kantharia, N. G., Rao, A. P., \& Sirothia, S. K. 2008, MNRAS, 383, 173

Kautsch, S. J., Gonzalez, A. H., Soto, C. A., et al. 2008, ApJ, 688, L5

Kormendy, J. 1985, ApJ, 295, 73

Kormendy, J., \& McClure, R. D. 1993, AJ, 105, 1793

Kormendy, J., Fisher, D. B., Cornell, M. E., \& Bender, R. 2009, ApJS, 182, 216 
Li, I. H., Yee, H. K. C., \& Ellingson, E. 2009, ApJ, 698, 83

Lin, D. N. C., \& Faber, S. M. 1983, ApJ, 266, L21

Lisker, T., Grebel, E. K., \& Binggeli, B. 2006, AJ, 132, 497

Lisker, T., Grebel, E. K., Binggeli, B., \& Glatt, K. 2007, ApJ, 660, 1186

Lisker, T., Grebel, E. K., \& Binggeli, B. 2008, AJ, 135, 380

Maltby, D. T., et al. 2009, submitted

Mastropietro, C., Moore, B., Mayer, L., et al. 2005, MNRAS, 364, 607

Mateo, M. L. 1998, ARA\&A, 36, 435

Matković, A., \& Guzmán, R. 2005, MNRAS, 362, 289

Matthews, L. D., Gallagher, J. S., III, Krist, J. E., et al. 1999, AJ, 118, 208

Mayer, L., Governato, F., Colpi, M., et al. 2001a, ApJ, 547, L123

Mayer, L., Governato, F., Colpi, M., et al. 2001b, ApJ, 559, 754

Michielsen, D., Boselli, A., Conselice, C. J., et al. 2008, MNRAS, 385, 1374

Misgeld, I., Mieske, S., \& Hilker, M. 2008, A\&A, 486, 697

Misgeld, I., Hilker, M., \& Mieske, S. 2009, A\&A, 496, 683

Moore, B., Lake, G., \& Katz, N. 1998, ApJ, 495, 139

Patterson, R. J., \& Thuan, T. X. 1996, ApJS, 107, 103

Pedraz, S., Gorgas, J., Cardiel, N., Sánchez-Blázquez, P., \& Guzmán, R. 2002, MNRAS, 332, L59

Peng, C. Y., Ho, L. C., Impey, C. D., \& Rix, H.-W. 2002, AJ, 124, 266

Phillips, A. C., Illingworth, G. D., MacKenty, J. W., \& Franx, M. 1996, AJ, 111, 1566

Rakos, K., \& Schombert, J. 2004, AJ, 127, 1502
Ryden, B. S., Terndrup, D. M., Pogge, R. W., \& Lauer, T. R. 1999, ApJ, 517, 650 Sabatini, S., Davies, J., Scaramella, R., et al. 2003, MNRAS, 341, 981

Sánchez-Janssen, R., Aguerri, J. A. L., \& Muñoz-Tuñón, C. 2008, ApJ, 679, L77

Sandage, A., \& Binggeli, B. 1984, AJ, 89, 919

Sandage, A., Binggeli, B., \& Tammann, G. A. 1985, AJ, 90, 1759

Schneider, S. E., Thuan, T. X., Magri, C., \& Wadiak, J. E. 1990, ApJS, 72, 245

Secker, J. 1996, ApJ, 469, L81

Smith, R. J., Lucey, J. R., Hudson, M. J., et al. 2009, MNRAS, 392, 1265

Smith Castelli, A. V., Bassino, L. P., Richtler, T., et al. 2008, MNRAS, 386, 2311

The, L. S., \& White, S. D. M. 1986, AJ, 92, 1248

Tonnesen, S., Bryan, G. L., \& van Gorkom, J. H. 2007, ApJ, 671, 1434

Trujillo, I., Erwin, P., Asensio Ramos, A., \& Graham, A. W. 2004, AJ, 127, 1917

Tully, R. B., \& Trentham, N. 2008, AJ, 135, 1488

van den Bergh, S. 1986, AJ, 91, 271

van Zee, L. 2001, AJ, 121, 2003

van Zee, L., Skillman, E. D., \& Haynes, M. P. 2004, AJ, 128, 121

Vollmer, B., Balkowski, C., Cayatte, V., van Driel, W., \& Huchtmeier, W. 2004, A\&A, 419, 35

Wirth, A., \& Gallagher, J. S., III 1984, ApJ, 282, 85

Wolf, C., Meisenheimer, K., Rix, H.-W., et al. 2003, A\&A, 401, 73

Wolf, C., Gray, M. E., \& Meisenheimer, K. 2005, A\&A, 443, 435

Wolf, C., Gray, M. E., Aragón-Salamanca, A., Lane, K. P., \& Meisenheimer, K. 2007, MNRAS, 376, L1 